\title{
EL SER Y EL TIEMPO. UNA POSIBILIDAD ONTOLÓGICA PARA LA TERAPIA OCUPACIONAL
}

\author{
BEING AND TIME. AN ONTOLOGICAL POSIBILITY FOR OCCUPATIONAL THERAPY
}

\section{Randy Yañez R. ${ }^{1}$, Eugenia Pizarro T. ${ }^{2}$.}

\begin{abstract}
Resumen
El presente trabajo se propone navegar en las posibilidades que otorgan las ideas sobre el Ser y el Tiempo desarrolladas por Martin Heidegger, como punto de partida para una reflexión ontológica sobre la ocupación, coherente con el ejercicio de la Terapia Ocupacional. Con este propósito, se revisan y contrastan conceptos centrales de la propuesta del autor alemán con el proyecto ampliamente aceptado por la comunidad de Terapeutas Ocupacionales, la naturaleza ocupacional del ser humano de Ann Wilcock. La discusión, además de centrarse en la invitación a los y las terapeutas ocupacionales a profundizar en este tema, se encuentra con una evidente sincronía entre el dasein hedeggeriano y la ocupación, aspectos que resuenan en la noción de becoming de Wilcock.
\end{abstract}

\section{Palabras clave:}

Ocupación, ontología, Heidegger, Wilcock, Tiempo.

\begin{abstract}
The present paper pretends to sail through the posibilities given by the notions about Being and Time developed by Martin Heidegger, as a starting point for an ontological reflection about occupation, coherent with the Occupational Therapy practice. For this purpose, core concepts of the german author are revised and contrasted with the project widely accepted in the occupational therapists community, the occupational nature of the human being by Ann Wilcock. The discussion, in addition to focusing on the invitation to the occupational therapists to go deeper into the topic, encounters an evident synchrony between Heidegger's dasein and occupation, both aspects that resonate in Wilcock's notion of becoming.
\end{abstract}

\section{Keywords:}

Occupation, ontology, Heidegger, Wilcock, Time.

1 Terapeuta Ocupacional, Magíster (c) en Bioética, Docente Instituto de Aparato Locomotor y Rehabilitación Universidad Austral de Chile, dirección postal: Campus Isla Teja s/n casa 2 A, fono: 63 - 2293510, correo electrónico: randyyanez@uach.cl

2 Terapeuta Ocupacional, Magíster en Comunicación, Docente Instituto de Aparato Locomotor y Rehabilitación Universidad Austral de Chile, dirección postal: Campus Isla Teja s/n casa 2 A, fono: 63 - 2293510, correo electrónico: eugeniapizarro@uach.cl 


\section{INTRODUCCIÓN}

"(...) porque el Dasein, entendido ontológicamente es sorge, cuidado. Puesto que al Dasein le pertenece por esencia el estar-en-el-mundo, su estar vuelto al mundo es esencialmente ocupación". (Heidegger, 2005, p.66)

Cuando el connotado filósofo alemán Martin Heidegger se pregunta por el sentido del Ser al inicio de su tratado "Ser y Tiempo" (en adelante SyT), no sólo establece que esta pregunta es la más universal de todas, sino que también es la más vacía (Heidegger, 2005). Con esto el autor pretende explicar que los esfuerzos realizados por la filosofía hasta ese momento habrían sido infructuosos a la hora de plantear y resolver las cuestiones ontológicas. Se hacía necesario entonces, establecer un nuevo esquema argumentativo que permitiera comprender la complejidad y la importancia que involucra este tipo de preguntas.

Con una evidente influencia por el existencialismo de la época y por la fenomenología que aprendiera de quien fue su primer maestro Edmund Husserl, Heidegger propone que la pregunta por el Ser está determinada por algo que hasta el momento ningún pensador había detenido en su profundidad: la temporalidad. A partir de esta premisa es que Heidegger realiza un cuestionamiento a la hasta entonces ontología basada en una conciencia a-temporal, sostenida principalmente por filósofos clásicos de la metafísica idealista como Aristóteles (2013), Descartes (2004) y en alguna medida, por Immanuel Kant ${ }^{3}$.

Si hacemos una breve revisión histórica de la Ciencia de la Ocupación, nos encontraremos con un patrón similar en las propuestas sobre ocupa-

3 Según Kant, en su Estética trascendental de la Crítica de la Razón Pura, la experiencia sensible es posible y válida gracias a los a priori de la sensibilidad. Kant llama a estos a priori de la sensibilidad las intuiciones o formas puras, que son el espacio y el tiempo. Su función sería actuar regulando a los materiales de la experiencia, para que puedan Ilegar a ser captados. En otras palabras, estas intuiciones puras otorgan a los materiales de la experiencia sensible la posibilidad de ser verdaderos objetos de la experiencia, o en sus propias palabras: fenómenos. ción que podríamos Ilamar "ontológicas" y que realizan autores considerados claves dentro de la disciplina. Grandes referentes de la Ciencia de la Ocupación y la Terapia Ocupacional, como Adolf Meyer (1977), Zemke y Clark (1996), Kielhofner (2004) y Townsend (1997), así como las revisiones que a estos autores se hace (Farnworth \& Fossey, 2003), coinciden en atribuirle a la ocupación un estatus de atemporalidad ${ }^{4}$ y por sobre todo, de externalidad al sujeto, es decir, un fenómeno que ocurre entre el sujeto individual y su medio ambiente. Son varios los ejemplos de modelos disciplinares en donde aparece nuevamente el mismo fenómeno de externalización del tiempo y de la ocupación, es decir, la ocupación como un producto ajeno a la esencia misma del Ser humano ${ }^{5}$.

Este tipo de análisis, si bien es cierto ha dado el sustento para la consolidación de la Ciencia de la Ocupación, adolece de no superar completamente la relación sujeto-objeto, dando una mirada mucho más profunda que la clásica, pero que aún parece ser insuficiente ${ }^{6}$. Si trasladamos este fenómeno al lenguaje heideggeriano, podríamos decir que todos estos análisis han quedado en el terreno óntico, es decir, en el terreno del ente que se relaciona con su

4 Atemporalidad en el sentido del tiempo heideggeriano. Esta temporalidad no tiene relación con la perspectiva histórica ni tampoco del tiempo como una categoría ajena o externa al sujeto, como se verá más adelante en el desarrollo de la idea del da-sein.

5 Ver por ejemplo la propuesta de Carrasco y Olivares publicada en 2009, en donde se ve a la ocupación como un fenómeno que ocurre en la intersección entre la persona, el ambiente y la actividad, y en donde la temporalidad ocurre luego de realizado este cruce, no siendo un determinante del mismo; en Kielhofner (2004) y su Modelo de Ocupación Humana, mantienen este patrón del sujeto y sus componentes fuera del ambiente dejando la temporalidad como consecuencia de su interacción. Townsend et al. (1997) en Modelo Canadiense de Desempeño Ocupacional incorpora a la persona inserta dentro de su ambiente pero a la vez separado de él, no desarrollando una clara idea sobre la temporalidad.

6 Comentario aparte merecen en nuestra opinión las propuestas de Michael Iwama (2006) y Claire Hocking (2011), cuyas aproximaciones a la ocupación tienen como punto de partida la narración del sujeto, abriendo el espacio para una comprensión compleja de este fenómeno. 
ambiente y no del Ser que de alguna manera "es" también su ambiente (Heidegger, 2005, p. 27).

Es indudable el aporte técnico, en términos prácticos e interventivos, que realiza el análisis del ente, sin embargo, la pregunta primera o fundamental pudiera ser sacada del terreno de los entes y planteada nuevamente desde un terreno ya ontológico: ¿Qué es la ocupación?, ¿Existe, efectivamente una ocupación?, ¿Es la ocupación algo ajeno, como objeto positivo ${ }^{7}$, a los seres humanos?, ¿Cual es el rol del tiempo en la ocupación?

Lejos de ser una materia novedosa, estas preguntas han tenido amplia discusión entre los y las cientistas ocupacionales, donde abundan las explicaciones fuertemente influenciadas por la época en las que fueron planteadas y por las diversas interpretaciones y aplicaciones que le hemos dado a esas teorizaciones en el terreno de la práctica. Una de estas respuestas/propuestas epistemológicas que ha cobrado fuerza en la actualidad está dirigida a comprender la relación que se establece entre la ocupación y los fenómenos sociales ${ }^{8}$. Con esta tendencia se refuerza un importante intento de des-medicalización de la disciplina - en el sentido de la exclusiva comprensión de los fenómenos desde una visión biomédica - que ha determinado en gran medida la forma en que se ha estudiado la ocupación hasta el momento (Fox, 2014).

Si tomáramos como válida esta forma de interpretación, en nuestra opinión, primero es necesario desprenderse de cualquier intento individualizante de situar a la ocupación, en términos ontológicos, en el ámbito de una elección aislada de la esencia humana, fuera de ella o distinta de lo que ser humano en sí significa. En este sentido, un Paradigma Social de la Ocupación así como cualquier ejercicio paradigmático en la Ciencia

7 Objeto positivo en el sentido de A. Comte en su Discurso sobre el espíritu positivo (1953)

8 Ver por ejemplo las propuestas realizadas por Simó y Kronenberg en Terapia Ocupacional sin Fronteras (2007), Magalhaes en Occupational Science: Society, Inclusion, participation (2012), Guajardo en Trujillo (2011), y Morrison \& Vidal en 2009, estos últimos con la propuesta de un Paradigma Social para la Ocupación Humana, entre otras. que sustenta nuestra disciplina, requiere superar la dualidad sujeto-ocupación - en tanto objeto positivo - dado que, de lo contrario, difícilmente podremos llegar a una comprensión más acabada de la Ocupación.

En esta empresa, la Ciencia de la Ocupación ha aceptado ciertas propuestas teóricas sin hacer un análisis profundo a sus bases filosóficas, perpetuando el sesgo histórico que ha tenido la visión binaria que se le da a la ocupación en nuestra profesión (Hammel, 2009). Para la superación de este sesgo, se requiere que dejemos de pensar por una parte en el sujeto como un objeto positivo, y por otra parte en la ocupación o el medioambiente también como objetos positivos, de manera de pasar a repensarlo no como elementos desagregados del sujeto sino que como elementos unificados y a la vez constitutivos del ser humano en su esencia.

A partir de esta dificultad es que, tal y como lo hizo Heidegger con la pregunta sobre el sentido del Ser, intentaremos defender la necesidad de profundizar en la pregunta ontológica por el sentido de la ocupación. Con este propósito, discutiremos por una parte la perspectiva de este autor y su reinterpretación ontológica del Ser y, por otra parte, una propuesta ampliamente aceptada en la comunidad de Terapeutas Ocupacionales, la naturaleza ocupacional del ser humano de Ann Wilcock (2006).

\section{El Ser y el tiempo en la filosofía de Martin Heidegger}

Al inicio de SyT, Heidegger parte con una alusión al sofista de Platón. Este inicio no es casual ya que el parágrafo citado revela el sentido trascendental de la pregunta por el Ser:

Porque manifiestamente vosotros estáis familiarizados desde hace mucho tiempo con lo que propiamente queréis decir cuando usáis la expresión "ente"; en cambio, nosotros creíamos otrora comprenderlo, pero ahora nos encontramos en aporía. (Platón, 1977, citado en Heidegger, 2005, p.23) 
Con esto Heidegger deja claro que las preguntas aparentemente sencillas, aquellas que nadie cuestiona, revisten una inusitada complejidad cuando se las vuelve a consultar de manera más profunda. Si planteamos por ejemplo la pregunta: ¿Cuál es el sentido de la Ocupación o de estar ocupado?, probablemente en sí misma no nos sorprenda y respondamos rápidamente a algo que parece obvio. Con mayor o menor complejidad iríamos tejiendo argumentación desde la Ciencia de la Ocupación y desde distintos autores para probablemente construir una definición con una marcada - y a la vez casi invisible - raíz biomédica en la forma de conocer y explicar dicho fenómeno. Pero, ¿Qué ocurre en términos ontológicos? El caso es que raramente nos preguntamos sobre la ocupación a este nivel, y cuando lo hemos hecho, hemos respondido sólo parcialmente. No se trata de volver estéril la pregunta ¿Qué es la Ocupación?, tampoco se trata de levantar una teoría universal sobre lo mismo, sino más bien se trata, al igual que lo hizo Heidegger con la pregunta por el Ser, de abrir interrogantes sobre aquello que no puede ni podría ser tematizado como un objeto o cosa.

Para comenzar a introducirnos en la filosofía heideggeriana y específicamente sobre la pregunta por el Ser, es necesario hacer algunas precisiones respecto de los conceptos nuevos que incorpora su filosofía, ya que muchas de las categorías propuestas por este autor y que utiliza ampliamente en sus explicaciones, no son las que comúnmente encontramos en el lenguaje cotidiano. Una de las características de esta filosofía es que se trata de una filosofía sui generis, por lo tanto las palabras y conceptos que utiliza son poseedores de esta misma particularidad.

\section{El Dasein o ser-ahí}

Para realizar el análisis sobre la pregunta del Ser, Heidegger propone una nueva categoría de análisis que debe desprenderse de lo estructural o esquemático, es decir, propone hacer un análisis existencial de un Ser en movimiento, un Ser que por sobre todo es movimiento y que transita en un contexto y tiempo específico, siendo esa su característica primordial. El estar en un tiempo determinado y en un lugar determinado de manera dinámica lo llamará Da-sein ó Ser-Ahí. ${ }^{9}$

Dasein es el término que Heidegger introduce para referirse también al "Ser que se pregunta por el ser", por ello el Dasein es exclusivamente el Ser del ente humano. Este Ser no se da aislado ni en el terreno de lo abstracto, sino que este Ser se da principalmente ocupado en su cotidianidad como veremos más adelante. El Dasein es entonces un ente privilegiado o el ente a partir del cual comienza su propia ontología, puesto que es el ente que tiene la pregunta y a la vez es el ente que lleva en sí mismo la respuesta. Dirá el mismo Heidegger (2005):

El Dasein no es tan sólo un ente que se presenta entre otros entes. Lo que lo caracteriza ónticamente es que a este ente le va en su ser este mismo ser. La constitución de ser del Dasein implica entonces que el Dasein tiene en su ser una relación de ser con su ser. Y esto significa, a su vez, que el Dasein se comprende en su ser de alguna manera y con algún grado de explicitud. Es propio de este ente el que con y por su ser éste se encuentre abierto para él mismo. (p. 22)

Así, este Dasein tendrá una propiedad que no podrá eludir, el tener-que-ser ${ }^{10}$. Esto significa que

9 El Dasein ó Ser-Ahí de Heidegger es un concepto que ha suscitado las más diversas investigaciones tanto en el campo de la filosofía como en el campo específico de algunas profesiones. En este caso, lo que nos importará para este trabajo será la idea del estar ocupado del Dasein durante su paso por la existencia, así como también el trato que se le dará a la dimensión de temporalidad o temporeidad.

10 Es necesario recordar que el estilo de escritura que utiliza Heidegger se caracteriza, entre otras cosas, por incorporar la creación de un nuevo campo semántico de las palabras. ej.: "tener-queser". Esto es para hacer notar en último término la unicidad e indivisibilidad que caracterizan las ideas que hay detrás, además de diferenciarlos de su acepción coloquial que, como mencionamos anteriormente, lo alejarían de su propuesta lingüística. Precisar también que la adecuación de los conceptos desde el original alemán a una interpretación en español ha sido siempre una empresa dificultosa que está en constante construcción. 
el Dasein no es sino que tiene que ser. El Dasein está de alguna forma obligado a ser, porque el Ser del Dasein no estará dado, ni predestinado, así como tampoco nadie hará al Dasein más que él mismo; es decir, el ser del Dasein dependerá de sí mismo. La importancia de esta aseveración, es que se deja atrás cualquier pretensión de explicación divina del Ser, determinada por un ser superior o por el destino. Este tener-que-ser también se relaciona con las ideas de libertad, voluntad o responsabilidad, que tendría un Dasein por definición incompleto y que se da a la tarea de transformarse en su camino de existencia al margen de cualquier determinismo. A este tenerque-ser, también Heidegger lo llamara "existir" (existenz), un existir no en su significación habitual o tradicional, sino que justamente tiene este sentido del tener-que-ser, de completarse y de transformarse.

\section{Ente o el mundo entitativo}

Ente es, según Heidegger, todo aquello que pensamos, todo lo que físicamente nos rodea, todo aquello de lo que hablamos, incluso nosotros mismos existen a nuestro alrededor una gran cantidad de cosas, algunas accesibles a la experiencia sensorial; así como otros entes abstractos a los cuales se accede a través de la razón. En palabras del mismo Heidegger (2005):

Pero llamamos "ente" a muchas cosas y en diversos sentidos. Ente es todo aquello de lo que hablamos, lo que mentamos, aqueIlo con respecto a lo cual nos comportamos de ésta o aquella manera; ente es también lo que nosotros mismos somos, y el modo como lo somos. (p. 17)

Cada cosa o cada clase de ente tiene también su propio modo de ser, siendo el modo de Ser del animal diferente al modo de ser de un objeto, por ejemplo, o el modo de ser de un ente abstracto, un número o palabra por ejemplo, muy distinto al modo de ser de una persona. No da lo mismo entonces cualquier ente al momento de intentar comprender el sentido del Ser, dirá Heidegger, sino que debemos comenzar a comprender el mundo desde aquel ente que se nos hace patente, sin falsificación, sin mediación. En este ente se debe leer el sentido del Ser, y desde aquel deberá arrancar el estudio: "A éste ente que somos en cada caso nosotros mismos, y que, entre otras cosas, tiene esa posibilidad de ser que es preguntar, lo designaremos con el término Dasein" (Heidegger, 2005, p. 18).

El ente privilegiado para comenzar toda ontología no es otro que cada uno de nosotros, es decir, nosotros mismos somos el ente más próximo, mi propio yo es el ente que me está más cercano. La importancia de esta diferenciación entre el terreno de lo entitativo y lo verdaderamente ontológico, radica en que los ejercicios disciplinares por desentrañar la esencia de la Ocupación, hasta el momento, han partido y se han explicado solo desde este terreno, careciendo aún de la posibilidad de un estudio ontológico de la ocupación humana como un suceso que le ocurre al Dasein y no sólo al ente humano.

\section{La Ocupación en Heidegger como un Estar-en-el-mundo}

Para ejercer su tener-que-ser entendido ahora como su completarse, hacerse y/o transformarse, el Dasein tiene una estructura que Heidegger denominará: estar-en-el-mundo. Este "estar" no obedece sólo al sentido de espacialidad que se le da, sino que también significará un estar en una cierta actividad, un hacer u obrar, un estar involucrado en cierto asunto, tal como ocurre cuando se está escribiendo, leyendo, realizando actividades de la vida diaria o de tiempo libre, es decir, en cierta ocupación ${ }^{11}$.

11 Este Estar-en-el-mundo reviste una complejidad lingüística. En alemán, al igual que en el Inglés, no existe diferencia entre el "ser" o "estar" ("sein" ó "being" respectivamente); por lo tanto este estar-en-el-mundo puede ser también un ser-en-el-mundo. Así, el "estar" perdería su sentido espacial para dar paso a su sig- 
El "mundo" de este estar-en-el-mundo, para Heidegger no es ningún ente físico o abstracto particular, ni tampoco un dominio particular del ente, sino que este mundo será el ámbito en el cual desarrollaremos nuestra existencia. Es "la apertura al ser" dirá el autor, lo que significa que la estructura del Dasein es esencialmente darse en un mundo. Estar-en-el-mundo es una estructura originaria y siempre total, pues no es posible que el Dasein no se dé en un ámbito. Con esta tesis se intenta superar la categoría binaria sujeto-ambiente a la que estamos acostumbrados y que encontramos en la mayoría de los trabajos y reflexiones disciplinares desde aquellos modelos teóricos que tradicionalmente sustentan nuestra práctica ${ }^{12}$.

estar-en-el-mundo es entonces estar involucrado o volcado a una cierta actividad, una actividad que es esencialmente práctica. Heidegger hablará entonces de un trato ${ }^{13}$ con las cosas, de un usar y manipular los entes. Estas cosas, tal como mencionábamos anteriormente y al igual que el Dasein, tienen su propio modo de ser. A estas cosas o entes que el Dasein usa en lo cotidiano, Heidegger las Ilamará entes intramundanos, útiles, o estar-a-la-mano (Zuhandenheit).

El ente intramundano será entonces aquel ente que es usado y manipulado por el Dasein, son los entes que entran en la actividad del Dasein y que comparecen cuando son usados, Heidegger hablará entonces de un trato con el ente intramundano ${ }^{14}$. Una idea central de esto, es que los entes intramundanos no pueden entenderse como sustancias ${ }^{15}$ en

nificación existencial. La separación entre el Ser y el Mundo será entonces para Heidegger meramente fenoménica y metodológica.

12 Podríamos nombrar como posible excepción al modelo Kawa de M. Iwama (2006), que integra al ser humano en su ambiente como un sujeto heterárquico con el resto de elementos, cuestionando de alguna forma el antropocentrismo teórico que presentan otros modelos teóricos para la práctica.

13 La expresión "trato", tiene el sentido de tratar con cosas en la experiencia, es decir, una experiencia de las cosas que se da en el trato mismo.

14 Mundano no solo en un sentido de un mundo "ambiental", sino que en el sentido de "apertura al ser"

15 Sustancia en su acepción aristotélica, para denotar el sentido del Ser que tendrían las cosas en sí, de su existencia. Recordemos que en Heidegger las cosas "son" pero no "existen". sí mismos, sino que ellos son en la medida en que están siendo usados, o dicho de otro modo, "su Ser consiste en ser usados". Así entonces, el útil (Zeug) comparece en la medida en que es utilizado. El modo de Ser del útil entonces, el estar-a-la-mano, es estar a disposición de comparecer ante el Dasein.

En términos de la relación que se da entre las cosas, Heidegger dirá que estos útiles remiten a otros útiles, - El lápiz remite al cuaderno y el cuaderno a la mesa, y la mesa a una habitación - Luego, los útiles también remiten a la materia prima, - El lápiz remite a la madera, que a su vez remite a la naturaleza, etc. Y por último, el útil también remitirá a la obra, es decir, al para qué; así, el lápiz remitirá al texto que se escriba con él, o el martillo remitirá a la casa que se ha construido con su comparecencia.

La obra también tiene el modo de ser del útil, porque es el Ser para qué de los útiles, es decir, la obra tiene una finalidad. Ciertamente la obra es un útil más, pero marca la finalidad de los útiles que se emplean en ella. Así, la obra en tanto útil también, remite a todos los útiles: las herramientas, la materia prima, la naturaleza, así como al portador y al usuario, o sea, a otro Dasein. En palabras del autor:

Aquello con lo que ante todo tiene que habérselas el trato cotidiano no son tampoco los utensilios, sino lo que primariamente nos ocupa y está por ende a la mano, es la obra misma, lo que en cada caso tiene que ser producido. Es la obra la portadora de la totalidad remisional dentro de la cual el útil comparece. (Heidegger, 2005, p. 78)

\section{El Dasein ocupándose y con otros: El ser- con-otros, estando-en-el-mundo}

El Dasein no es sólo estar-en-el-mundo, sino también un ser con otros o ser-entre-otros. No es posible entender al Dasein sino en la medida 
en que está con otros; sólo así se encuentra a sí mismo. Por ello, Heidegger instalará el concepto de coexistencia (Mitdasein). La coexistencia para el autor será una ocupación en común, ya que la existencia no es una actividad aislada en medio de otras actividades aisladas. Nuestra propia existencia es una actividad en común y a causa de ello, no sería posible prescindir esencialmente del otro. El Dasein se encuentra inmediatamente a sí mismo en la actividad que realiza, y eso que realiza lo realiza con otros. Por lo tanto, el Dasein que comparece es el Dasein coexistente:

Esta coexistencia de los otros queda intramundanamente abierta para un Dasein y así también para los coexistentes, tan sólo porque el Dasein es en sí mismo esencialmente coestar (...) el coestar determina existencialmente al Dasein incluso cuando no hay otro que esté fácticamente ahí, y que sea percibido. También el estar solo del Dasein es un coestar en el mundo (...) el coestar y la facticidad del convivir no se funda, entonces, en un encontrarse juntos de varios "sujetos". (Heidegger, 2005, p. 125)

Según el autor, todo este mundo de ocupaciones no se comprende si no está sostenido por varios Dasein. El sentido de este programa existencial ocurre debido a que hay otros Dasein con los cuales coexistir, con los cuales co-ocuparse ya que este Dasein es eminentemente actividad, no es primariamente un ser pensante, - como lo diría Descartes -. Este Ser no se da primariamente en el plano de la conciencia sino que en el plano de la acción, el ser pensante cartesiano no manipula ni usa cosas, es decir, no es un ser ocupado.

Así, el Dasein es el ente cotidiano, es el ente evidente al cual es posible acceder pero sólo en tanto está en actividad en el mundo, en tanto trata con los útiles, en tanto relacionado con otros Dasein mediante la obra. Dicha obra, al igual que este Dasein ocupado, será evidente. Así, lo primario y patente no será un yo aislado - sólo pensante -, desvinculado, sino un yo en la obra, usando y manipulando utensilios, en conjunto con otros. La estructura del Dasein no es solamente un estar-enel-mundo, sino también un estar-entre-otros en el mundo, en una ocupación en común.

\section{La propuesta sobre el Ser Ocupacional y el Tiempo en Wilcock.}

La terapeuta ocupacional Ann Wilcock (2006), aborda el tema del Ser en el contexto de una propuesta que explica la salud desde una perspectiva ocupacional, bajo una concepción evolutiva de la relación ocupación - salud ${ }^{16}$. Esta ocupación se fundamenta en la noción de una "naturaleza ocupacional del ser humano", la que conlleva implicancias no sólo para la comprensión del concepto de salud, sino que por sobre todo para la forma en que la Ciencia de la Ocupación puede llegar a entender la idea de "Ser".

Para propósitos de la presente discusión, nos detendremos en tres aspectos de la propuesta de Wilcock; la perspectiva evolucionista, el ser a través del hacer y la idea de becoming.

En primer lugar, bajo la perspectiva evolucionista de la ocupación, las transformaciones de la especie, desde el homo habilis hasta nuestros días, estarían en directa relación con las ocupaciones que ha desarrollado el ser humano, esto se entiende como un proceso de retroalimentación; la ocupación ha propiciado y dado forma a la evolución de aspectos anatómicos evidentes y a la especialización cerebral, que a su vez, han potenciado el despliegue de nuevas y variadas ocupaciones.

Este proceso habría conducido el desarrollo de un repertorio de capacidades como especie, que a nivel individual constituyen un potencial innato y que se encuentran a la base del hacer humano, lo que permite la satisfacción de necesidades para la sobrevida y el bienestar. Así, las capacidades

16 Nos centraremos en el primer concepto de esta relación, sin detenernos en el segundo - la salud - cuyo análisis escapa a los propósitos de este trabajo. 
son un requisito para la ocupación humana y una necesidad en sí mismas. Estas capacidades deben ser utilizadas ya que el cerebro necesita nuevos desafíos, de lo contrario, se espera un impacto negativo en la salud.

Esta perspectiva supone una forma de entender a la especie humana en un marco temporal que implica transformación. En este sentido, se vislumbran en la propuesta de Wilcock dos tipos de evolución: la primera, como se describió previamente, es biológica y más lenta; no encontrándose cambios apreciables desde los primeros homo sapiens hasta nuestros días. La segunda es ocupacional, relacionada con procesos culturales y sociales. Esta última es identificable a través de la historia y se reconocen en ella cambios en la forma de usar el tiempo y en el sentido del hacer, que atraviesan y configuran - o son configurados por - la estructura social, los avances tecnológicos y la relación con la naturaleza.

Al respecto, Wilcock organiza los cambios identificables en eras ocupacionales que transcurren desde los cazadores - recolectores hasta la era postindustrial. Cabe destacar que la transformación ocupacional no es descrita como un fenómeno lineal, destacándose la existencia paralela de distintos estilos de vida, relacionados a diversas eras; al respecto se indica como ejemplo la presencia actual de pueblos cazadores recolectores.

Por otra parte, Wilcock (2006) propone entender la ocupación humana a partir de la tríada hacer (doing) - ser (being) - becoming ${ }^{17}$. A continuación nos detendremos en la relación ser - hacer.

Wilcock presenta como primer paso la idea del hacer, la cual relaciona a la satisfacción de necesidades y al uso de las capacidades; en este sentido, este sería el aspecto de la ocupación más cercano a la biología y la comprensión evolucionista del ser

17 Utilizaremos el concepto becoming en su idioma original. Cabe mencionar que este ha sido traducido como "llegar a ser" (Kronenberg, Simó Algado y Pollard, 2007; Rubio Ortega y Sanz Valer, 2011), lo que debido al énfasis que esta traducción pone en la meta, finalidad, o término de un proceso, tendería a distorsionar la noción propuesta por la autora. humano. Como segundo paso, se aborda el concepto de Ser como algo difícil de describir, aproximándose a su definición a través de menciones a Hegel y al mismo Heidegger, para luego detenerse en la idea de autoactualización e identidad de Maslow. Esta última referencia a la idea del Ser, en nuestra opinión, sentará las bases en la teoría de la autora para la construcción de los aspectos centrales de su perspectiva ocupacional de la salud. Al respecto, el ser es entendido en relación al hacer, presentándose esta idea como el ser a través del hacer.

La noción del ser a través del hacer o hacer para ser, sugiere interconexión de ambos aspectos que se comprenden indivisibles a la hora de analizar la salud desde una perspectiva ocupacional. Esta díada se relaciona, además, con las nociones de identidad, libertad y creatividad, entre otras. Bajo esta clave, el bienestar sólo pueda alcanzarse si en el hacer están presentes el significado, el propósito y la capacidad de elegir. En este contexto, se encuentra nuevamente una nota evolucionista, que sugiere que "el uso del tiempo significativo y con propósito es parte de nuestra herencia biológica" (Wilcock, 2006, p.116).

A esto se incorpora el tercer aspecto de la tríada, el concepto de becoming. Este introduce de manera más evidente la noción de tiempo, entendiéndose en términos generales como un proceso de ser en el hacer. Se define, además, como llegar a ser. Al respecto, identificamos tres aspectos que configuran la idea de becoming que, en nuestra opinión, es necesario distinguir.

En primer lugar, la noción de tiempo y de la inevitabilidad del cambio, ya que las personas siempre están becoming algo diferente, medie o no la voluntad de hacerlo. En segundo lugar, el becoming como proceso incompleto, nunca terminado o terminable; al respecto se enfatiza el transcurso del hacer por sobre el resultado. Por último, la connotación valórica del becoming, en nuestra opinión identificable en la cercanía a la idea de auto-actualización. En este sentido, se entiende becoming como despliegue de potencialidades, un alcance del nivel más alto de desarrollo personal y transcendencia. 
La comprensión de este ser en clave autoactualización y el becoming como este Ser en el tiempo, reflejan diferencias importantes con la perspectiva de Heidegger. Será el ser en el hacer, el punto de intersección más interesante de ambas teorías, pudiendo tener implicancias para la Ciencia de la Ocupación que consideramos relevantes de incorporar.

\section{DISCUSIÓN}

La tesis heideggeriana en Ser y Tiempo presenta evidentes similitudes con algunos aspectos de la Terapia Ocupacional y la Ciencia de la Ocupación. Esta relación, pocas veces profundizada y francamente omitida por autores y autoras disciplinares, nos invita a ver al ser humano como un ente que ontológicamente se ocupa, cuyo sentido de ser más profundo tendría que ver con el estar ocupado y el estar en relación con otros a través del tiempo.

Así, en un sentido ontológico, la Ocupación Humana como la entendemos quienes pensamos y practicamos la Terapia Ocupacional, encuentra en Heidegger su estado más puro, evidenciando que el estar ocupado no sería otra cosa que la vida misma. Si acordáramos como disciplina que nuestro objeto de estudio es entonces la Ocupación Humana, a la luz de esta teoría, nuestro objeto de estudio sería la completa existencia humana, en donde la relación entre salud y ocupación no sería más que sólo uno de los aspectos de interés disciplinar.

En este mismo sentido y como expusimos anteriormente, la propuesta del Ser de Wilcock pierde fuerza en términos ontológicos al estar centrada en elementos biologicistas, salubristas y evolucionistas. Al respecto, el tener-que-ser de Heidegger se encontraría en tensión con este acento, en cierto sentido determinista, de Wilcock. Más que tratarse de un proyecto existencial sobre la Ocupación Humana, la autora trata de otorgar los fundamentos desde las ciencias biomédicas al fenómeno de la ocupación y su relación con la sa- lud. La vinculación más patente entre la propuesta de Heidegger y Wilcock no se encuentra entonces en la idea de Ser, sino que en la idea del tiempo. En este sentido, el becoming se acerca al existir (existenz) heideggeriano en su integración de serhacer-tiempo.

Otro punto que nos parece relevante, es que la propuesta de Heidegger encontrará un segundo momento con Emanuel Lévinas (2003, 2006), quien profundiza en la idea de la existencia de un otro - al que llamará alteridad - como un elemento fundamental para completar el estudio de la ontología del Ser. Lévinas repara en que este ser no puede ser si no se proyecta a otros, es decir, trata de la trascendencia de un Ocupar-se-con-otros. Bajo esta perspectiva, las co-ocupaciones de las cuales se habla actualmente en Ciencia de la Ocupación (Zemke \& Clark, 1996; Pierce, 2003; Olson, 2003, citados en Pickens \& Pizur-Barnekow, 2009) no se levantarían como una categoría distinta, sino que nuevamente reflejan el sentido más esencial del "Ser-ocupado" en sí.

Tal y como Heidegger se dio a la tarea de revisar y cuestionar los proyectos ontológicos realizados hasta ese entonces, creemos que sería pertinente plantearse esta misma tarea para la Ocupación Humana. Las distintas teorías disciplinares hasta el momento no han logrado profundizar en esta temática, siendo a nuestro juicio un ámbito inconcluso que ha enfatizado en la comprensión cartesiana del fenómeno de la ocupación, dejando un vacío que no favorece la construcción de nuevos conocimientos desde distintas perspectivas. Pensamos que, por ejemplo, el antes mencionado Paradigma social de la ocupación puede ser revisado a la luz de la tesis de la alteridad levinasiana de manera de poder integrarse con la idea de las $\mathrm{CO}^{-}$ ocupaciones a partir de una misma raíz ontológica.

La invitación es entonces a reflexionar en torno dicha raíz ontológica, a investigar en las bases filosóficas de nuestra disciplina de manera de enriquecer los cimientos sobre los cuales se edifica la Ciencia de la Ocupación. Creemos que con la propuesta heideggeriana sobre el Ser y el tiempo, 
podemos en parte, dar sustento a lo que en la práctica de la Terapia Ocupacional muchas veces asoma como intuición. Desde el ámbito disciplinar, Ann Wilcock abre la senda para esta discusión, sin embargo, la tarea está aún lejos de ser concluida, dejando a los y las terapeutas ocupacionales con la obligación de reflexionar y debatir sobre la complejidad que reviste la Ocupación Humana.

\section{BiBLIOGRAFÍA}

Aristóteles. (2013). Metafísica (5a. ed.). Madrid: Gredos.

Carrasco Madariaga, J. \& Olivares Aising, D. (2009). Haciendo camino al andar: construcción y comprensión de la ocupación para la investigación y práctica de la Terapia Ocupacional. Revista Chilena de Terapia Ocupacional, 0(8). doi:10.5354/07176767.2008.55

Comte, A. (1953). Discurso sobre el espíritu positivo. Buenos Aires: Aguilar.

Descartes, R. (2004). Discurso del método: meditaciones metafísicas. Buenos Aires: Gradifco.

Farnworth, L., \& Fossey, E. (2003). Occupational Terminology Interactive Dialogue. Journal of Occupational Science, 10(3), 150153. doi:10.1080/14427591.2003.9686523

Fox, V. (2014). Water: A Useful Metaphor for Occupation. Journal of Occupational Science, 1-11. doi:10.1080/14427591.2014.8 97203

Hammell, K. W. (2009). Sacred Texts: A Sceptical Exploration of the Assumptions Underpinning Theories of Occupation. Canadian Journal of Occupational Therapy, 76(1), 6-13. doi:10.1177/000841740907600105

Heidegger, M. (2005). Ser y tiempo (4a. ed.). Santiago: Universitaria.

Hocking, C. (2011). The challenge of occupation. Describing the things people do. Journal of Occupational Science, 16(3), 140150. doi: 10.1080/14427591.2009.9686655

Iwama, M. (2006). The kawa model. Philadelphia: Churchill Livingstone Elsevier
Kant, I. (2006). Crítica de la razón pura. México D.F: Taurus.

Kielhofner, G. (2004). Terapia Ocupacional. Modelo de ocupación humana. Teoría y aplicación (3a. Ed.). Panamericana.

Kronenberg, F., Simó Algado, S. \& Pollard, N. (2007). Terapia Ocupacional sin fronteras. Aprendiendo del espritu de supervivientes. Buenos Aires: Panamericana.

Levinas, E. (2003). De otro modo que ser o más allá de la esencia. Salamanca: Ediciones Sígueme.

Levinas, E. (2006). Totalidad e infinito: ensayos sobre la exterioridad (7a. ed.). Salamanca: Ediciones Sígueme.

Meyer, A. (1977). The philosophy of occupational therapy. American Journal of Occupational therapy, 31(10), 639-642. (Original de 1922)

Molke, Daniel K., Deborah Laliberte-Rudman, \& Helene J. Polatajko. (2004). The promise of occupational science: A developmental assessment of an emerging academic discipline. Canadian Journal of Occupational Therapy, 71(5): 269-280. doi: 10.1177/000841740407100505

Morrison, R. \& Vidal, D. (2009). Análisis ontológico de la ocupación humana. (Tesis de grado, Universidad Austral de Chile). Recuperado de cybertesis.uach.cl/tesis/uach/2009/fmv648a/doc/fmv648a.pdf

Pickens, N. D., \& Pizur-Barnekow, K. (2009). Co-occupation: Extending the dialogue. Journal of Occupational Science, 16(3), 151156. doi:10.1080/14427591.2009.9686656

Rubio Ortega, C., Sanz Valer, P. (2011). Reflexiones acerca de hacer, ser y llegar a ser. [Traducción]. TOG (A Coruña), 8(14), 1 - 30. Recuperado de_http://www.revistatog.com/num14/pdfs/historia2.pdf

Trujillo Rojas. (2011) Ocupación: sentido, realizacion y libertad: Diálogos ocupacionales en torno al sujeto, la sociedad y el medio ambiente. Bogotá: Universidad Nacional de Colombia.

Townsend, E. (1997). Enabling occupation. an Occupational Therapy perspective. Otawa: CAOT Publications.

Wilcock, A. (2006). An occupational perspective of health. Thorofare: Slack.

Wilcock, A. (2007). Occupation and health: Are They One and the Same?, Journal of Occupational Science, 14:1, 3-8, DOI: 10.1080/14427591.2007.9686577

Whiteford, G., \& Hocking, C. (2012). Occupational Science: society, inclusion, participation. UK, Wiley-Blackwell. 